\title{
Regulation of Cyclooxygenase 2 by Filifactor alocis in Fibroblastic and Monocytic Cells
}

\author{
Marjan Nokhbehsaim $\mathbb{D}^{1},{ }^{1}$ Andressa V. B. Nogueira $\mathbb{D}^{2},{ }^{2}$ Sandor Nietzsche, ${ }^{3}$ Sigrun Eick $\mathbb{D}^{4}{ }^{4}$ \\ and James Deschner iD ${ }^{2}$ \\ ${ }^{1}$ Section of Experimental Dento-Maxillo-Facial Medicine, Center of Dento-Maxillo-Facial Medicine, University of Bonn, \\ Bonn, Germany \\ ${ }^{2}$ Department of Periodontology and Operative Dentistry, University Medical Center of the Johannes Gutenberg University, \\ Mainz, Germany \\ ${ }^{3}$ Center of Electron Microscopy, University Hospital Jena, Jena, Germany \\ ${ }^{4}$ Department of Periodontology, Laboratory of Oral Microbiology, University of Bern, Bern, Switzerland
}

Correspondence should be addressed to Andressa V. B. Nogueira; a.nogueira@uni-mainz.de

Received 17 June 2019; Revised 14 November 2019; Accepted 6 December 2019; Published 3 February 2020

Academic Editor: Kutty Selva Nandakumar

Copyright (C) 2020 Marjan Nokhbehsaim et al. This is an open access article distributed under the Creative Commons Attribution License, which permits unrestricted use, distribution, and reproduction in any medium, provided the original work is properly cited.

\begin{abstract}
Periodontitis is a prevalent chronic inflammatory disease triggered by a synergistic and dysbiotic microbiota present in the oral biofilm. This in vitro study is aimed at evaluating the regulation of cyclooxygenase (COX) 2 expression and production by the periodontopathogen Filifactor alocis in human gingival fibroblastic (HGF-1) and monocytic (THP-1) cells and also at investigating the underlying cellular pathway mechanisms. HGF-1 and THP-1 cells were exposed either to F. alocis or to the proinflammatory cytokine tumor necrosis factor alpha (TNF $\alpha$ ) for 1 and $2 \mathrm{~d}$ to examine the COX2 expression by qPCR. COX2 protein levels were evaluated by ELISA in $F$. alocis-stimulated cells. Both types of cells were also stimulated with a blocking tolllike receptor (TLR)2 antibody or specific inhibitors against MAPKs. F. alocis significantly $(p<0.05)$ increased COX2 at both transcriptional and protein levels in both HGF-1 and THP-1 cells. Moreover, the stimulatory effect of F. alocis on COX2 was more pronounced in HGF-1 cells in comparison to THP-1 cells. F. alocis upregulated the COX2 expression in a dose-dependent manner in both type cells at $1 \mathrm{~d}$. TNF $\alpha$ also significantly $(p<0.05)$ increased the COX2 expression in both cells. After preincubation of HGF-1 and THP-1 cells either with a neutralizing anti-TLR2 antibody or with specific MAPK inhibitors, the $F$. alocis-upregulated COX2 expression was significantly $(p<0.05)$ suppressed at $1 \mathrm{~d}$. Our in vitro study provides original evidence that $F$. alocis stimulates COX2 production in fibroblastic and monocytic cells through TLR2 and MAPK mechanisms, suggesting a role of this periodontopathogen in the etiopathogenesis of periodontitis.
\end{abstract}

\section{Introduction}

Periodontitis is a chronic inflammatory disease triggered by a synergistic and dysbiotic microbiota present in the oral biofilm. Periodontopathogenic bacteria and their products activate the host immune response leading to an overproduction of proinflammatory mediators. The progression of periodontitis is associated with a sustained and excessive immune-inflammatory response resulting in irreversible destruction of the periodontal supporting tissues and, eventually, in tooth loss $[1,2]$. In the periodontal tissues, both res- ident and infiltrating immune cells such as fibroblasts, monocytes, and macrophages are responsible for the increase in proinflammatory mediators like interleukin- (IL-) $1 \beta$, tumor necrosis factor alpha (TNF $\alpha)$, cyclooxygenase (COX)2, and matrix metalloproteinases. These mediators play an important role in the hard and soft tissue destruction by modulating the inflammatory cascade in periodontitis [3].

COX exists in two isoforms: COX1 and COX2. COX1 is constitutively expressed in many cells and tissues and is mainly involved in the maintenance of tissue homeostasis [4]. In contrast, COX2 is an inducible enzyme expressed by 
cells during inflammatory processes [4]. COX2 is induced by proinflammatory and infectious agents and is responsible for the conversion of arachidonic acid into prostaglandin (PG)E2 [5], which is actively involved in tissue damages by changing the connective tissue metabolism and by enhancing osteoclastic bone resorption, showing an evident association with the tissue destruction during periodontal disease progression $[6,7]$. COX2 plays an important role in the inflammatory process in periodontal diseases. Increased levels of COX2 and PGE2 have been demonstrated in saliva, gingiva, and gingival crevicular fluid of patients with periodontal disease [8-13].

Some studies have shown a strong association between Filifactor alocis and periodontitis $[14,15]$. This periodontopathogen is a Gram-positive anaerobic bacterium that has been detected in a higher number in saliva as well as in supragingival and subgingival biofilm of periodontal patients as compared to healthy subjects [15-18]. F. alocis has a high prevalence in periodontitis that could be attributed to its ability to invade periodontal cells and tissues, to resist oxidative stress and to stimulate, alone or together with periodontal pathogens, the secretion of proinflammatory cytokines $[19,20]$. Although $F$. alocis has been associated with periodontitis, no previous study has examined whether this periodontopathogen modulates the expression of COX2 by professional and accessory immune cells. Thus, the aim of the present study was to evaluate the effect of F. alocis on the COX2 expression and production by monocytic and fibroblastic cells and to unravel the underlying pathway mechanisms.

\section{Materials and Methods}

2.1. Culture and Treatment of Cells. Human gingival fibroblast cell line (HGF-1) (ATCC ${ }^{\circledR}$ CRL-2014 ${ }^{\mathrm{TM}}$, LGC Standards, Wesel, Germany) and human acute monocytic leukemia cell line (THP-1) (CLS Cell Lines Service, Eppelheim, Germany) were used. HGF-1 was seeded on 6-well cell culture plates $\left(5 \times 10^{4}\right.$ cells/well $)$ and grown to $80 \%$ confluence in Dulbecco's minimal essential medium (DMEM, Invitrogen, Karlsruhe, Germany) supplemented with $10 \%$ fetal bovine serum (FBS, Invitrogen), 100 units penicillin, and $100 \mu \mathrm{g} / \mathrm{ml}$ streptomycin (Invitrogen). THP-1 was seeded once their concentration reached $1 \times 10^{6}$ cells/ml using RPMI 1640 medium (Invitrogen) supplemented with $10 \%$ FBS, 100 units penicillin, and $100 \mu \mathrm{g} / \mathrm{ml}$ streptomycin. Both types of cells were grown at $37^{\circ} \mathrm{C}$ in a humidified atmosphere of $5 \% \mathrm{CO}_{2}$, and every other day, the cell culture medium was changed. One day prior to the experiments, the FBS concentration was reduced to $1 \%$.

The oral pathogenic bacterium F. alocis ATCC $35896^{\mathrm{TM}}$ was used to stimulate the cells at different concentrations (optical density (OD): 0.05, 0.1, and 0.2) as in our previous study [21]. F. alocis was precultivated in an anaerobic atmosphere on Schaedler agar plates (Oxoid, Basingstoke, UK) for $48 \mathrm{~h}$ and, then, suspended in phosphate-buffered saline $\left(\mathrm{OD}_{660 \mathrm{~nm}}=1\right.$, equivalent to $1.2 \times 10^{9}$ bacterial cells $\left./ \mathrm{ml}\right)$. Subsequently, the bacteria suspension was exposed twice to ultrasonication (160 W for $15 \mathrm{~min}$ ) resulting in total bacterial killing. In order to unravel intracellular signaling mechanisms by which $F$. alocis could possibly modulate COX2 expression, cells were preincubated with specific inhibitors of different pathways (MEK1 and MEK2: U0126, $10 \mu \mathrm{M}$; P38: SB203580, $10 \mu \mathrm{M}$; pyrrolidine dithiocarbamate: PDTC, $10 \mu \mathrm{M}$; and JNK inhibitor II: SP600125, $10 \mu \mathrm{M}$; all from Calbiochem, San Diego, CA, USA), $1 \mathrm{~h}$ prior to the F. alocis stimulation. Furthermore, cells were also preincubated with a blocking anti-human toll-like receptor (TLR) 2 monoclonal antibody $(10 \mu \mathrm{g} / \mathrm{ml}$; eBioscience, San Diego, CA, USA) $45 \mathrm{~min}$ prior to the $F$. alocis stimulation. Moreover, in order to mimic inflammatory conditions in vitro, cells were treated with human recombinant tumor necrosis factor (TNF) $\alpha$ (Biomol, Hamburg, Germany), which is one of the key inflammatory mediators in the pathogenesis of the periodontal diseases. TNF $\alpha$ was applied at a concentration of $1 \mathrm{ng} / \mathrm{ml}$, which is in the range of levels usually found in the gingival crevicular fluid (GCF) of periodontally diseased patients and which has been used by other investigators $[22,23]$. Untreated cells served as a control.

2.2. Scanning Electron Microscopy. Scanning electron microscopy images were taken to visualize the attachment of $F$. alocis ATCC 35896 on fibroblasts. The cells were cultured on glass slides until a monolayer was formed and thereafter exposed to $F$. alocis for $1 \mathrm{~h}$. Samples were fixed with $2 \%$ glutaraldehyde in $0.1 \mathrm{M}$ cacodylate buffer for $30 \mathrm{~min}$, washed twice with cacodylate buffer, and dehydrated using a $10 \%$ graded ethanol series $(10 \mathrm{~min}$ each concentration). At the Center of Electron Microscopy, University Hospital of Jena, Germany, critical point drying was performed, and samples were sputter-coated with gold and examined with a ZEISS LEO-1530 Gemini (Carl Zeiss NTS, Oberkochen, Germany) equipped with a field emission electron gun at $10 \mathrm{keV}$.

2.3. Real-Time PCR. Total RNA was extracted by using a RNA extraction kit (RNeasy Mini Kit, Qiagen, Hilden, Germany) following the manufacturer's protocol. Subsequently, RNA concentration was verified by a spectrophotometer (NanoDrop ND-2000, Thermo Fisher Scientific, Wilmington, DE, USA), and 500 ng of total RNA was transcribed to cDNA using the iScript ${ }^{\mathrm{TM}}$ Select cDNA Synthesis Kit (Bio-Rad Laboratories, Munich, Germany) at $42^{\circ} \mathrm{C}$ for $90 \mathrm{~min}$ followed by $85^{\circ} \mathrm{C}$ for $5 \mathrm{~min}$, according to the manufacturer's instructions. The gene expression of COX2 and glyceraldehyde-3-phosphate dehydrogenase (GAPDH) as an endogenous control was evaluated by quantitative RTPCR by using an iCycler iQ5 Real-Time PCR Detection System (Bio-Rad). A $25 \mu \mathrm{l}$ PCR reaction mixture was prepared containing $1 \mu \mathrm{l}$ of cDNA, $12.5 \mu \mathrm{l}$ of SsoAdvanced ${ }^{\mathrm{TM}}$ Universal SYBR $^{\circledR}$ Green Supermix (Bio-Rad), $2.5 \mu$ l of primers, and $9 \mu \mathrm{l}$ RNase-free water. The thermal protocol of the PCR reaction was $95^{\circ} \mathrm{C}$ for $5 \mathrm{~min}$, followed by 40 cycles of denaturation at $95^{\circ} \mathrm{C}$ for $10 \mathrm{~s}$ and combined annealing/extension at $60^{\circ} \mathrm{C}$ for $30 \mathrm{~s}$. This analysis was performed in triplicate. The data analysis of gene expression was performed using the comparative threshold cycle (CT) method. 


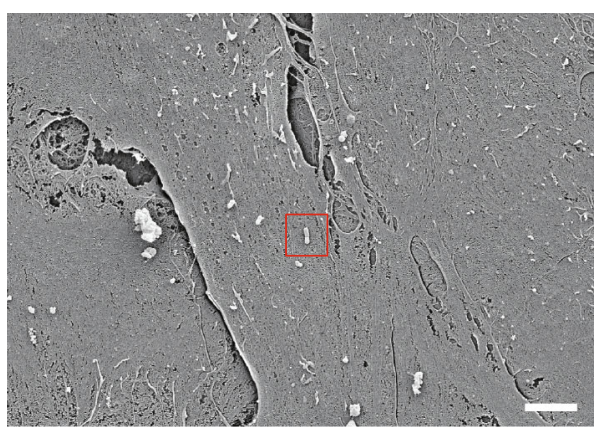

(a)

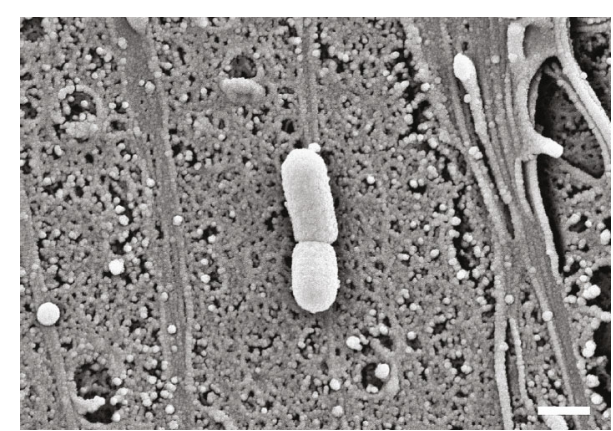

(b)

Figure 1: (a, b) Scanning electron microscopy images of F. alocis attached to fibroblastic cells. Scale bars: $5 \mu \mathrm{m}$ (a) and $500 \mathrm{~nm}(\mathrm{~b})$.

2.4. ELISA. HGF-1 or THP-1 cell lysates were used to evaluate the levels of COX2 by using commercially available sandwich enzyme-linked immunoassay (ELISA) detection kit (DYC4198-2; human/mouse Total COX2 ELISA with DuoSet, R\&D Systems Europe, Abingdon, United Kingdom) according to the manufacturer's instructions. The COX2 concentration was measured by spectrophotometry using a microplate reader (PowerWave $\mathrm{X}$, BioTek Instruments, Winooski, VT, USA) at $450 \mathrm{~nm}$ with wavelength correction at $540 \mathrm{~nm}$. Total protein concentration was measured using Pierce BCA Protein Assay Kit (23227, Thermo Fisher Scientific, Pierce Biotechnology, Rockford, USA) and used to normalize the data.

2.5. Statistical Analysis. Statistical analysis was performed using the software IBM SPSS Statistics 22. The mean values and standard errors of the mean (SEM) were calculated. For statistical analysis, parametric (ANOVA followed by post hoc Dunnett's and Tukey's tests) and nonparametric tests (Wilcoxon and Mann-Whitney $U$ tests) were used. Significant differences between groups were considered at $p$ value $<0.05$. All experiments were performed in triplicate and repeated at least twice.

\section{Results}

3.1. F. alocis Accumulation on Gingival Fibroblast. Figures 1(a) and 2(b) show F. alocis and its capability to attach to periodontal fibroblastic cells.

3.2. Regulation of COX2 by F. alocis in HGF-1 Cells. First, we investigated the regulation of COX2 in the presence and absence of the periodontopathogen $F$. alocis in HGF-1 cells. As shown in Figure 2(a), F. alocis caused a significant $(p<0.05)$ increase in the constitutive COX2 expression at 1 and $2 \mathrm{~d}$, as analyzed by qPCR. Furthermore, the stimulatory effect of $F$. alocis on the COX2 expression was dose-dependent at $\mathrm{d}$, with the highest COX2 expression at the highest concentration of $F$. alocis (Figure 2(b)). The stimulatory action of $F$. alocis on COX2 was also found at the protein level at $1 \mathrm{~d}$. COX2 protein levels were significantly $(p<0.05)$ higher in cell lysates from $F$. alocis-stimulated HGF-1 cells as compared to unstimulated control cells, as determined by ELISA
(Figure 2(c)). Interestingly, the proinflammatory cytokine TNF $\alpha$ also significantly $(p<0.05)$ increased the COX2 expression in HGF-1 cells at $1 \mathrm{~d}$ and $2 \mathrm{~d}$ (Figure 2(d)).

3.3. Regulation of COX2 by F. alocis in THP-1 Cells. Next, we sought to evaluate the influence of $F$. alocis on COX2 expression and protein levels in THP-1 cells. Like in HGF-1 cells, $F$. alocis caused a significant $(p<0.05)$ upregulation of COX 2 in THP- 1 cells at $1 \mathrm{~d}$ and $2 \mathrm{~d}$, as demonstrated in Figure 3(a). Moreover, the upregulation of COX2 expression by F. alocis was also dose-dependent in THP-1 cells. Again, the highest COX2 expression was observed at the highest concentration of $F$. alocis, and the protein levels were also paralleled by the gene expression levels of COX2 (Figures 3(b) and 3(c)). Following stimulation with TNF $\alpha$, THP-1 cells showed a significantly $(p<0.05)$ increased COX2 expression at $1 \mathrm{~d}$ but not $2 \mathrm{~d}$ (Figure $3(\mathrm{~d})$ ).

3.4. Involvement of TLR2 and Signaling Pathways in the Actions of F. alocis. Next, we sought to elucidate whether the stimulatory effects of $F$. alocis on COX2 expression were mediated through TLR2. Interestingly, when HGF-1 and THP-1 cells were preincubated with an anti-TLR2 blocking antibody, the stimulatory effect of $F$. alocis on COX2 was significantly $(p<0.05)$ abolished at $1 \mathrm{~d}$, as shown in Figures 4(a) and 4(b). Furthermore, we examined the involvement of intracellular pathways typically associated with inflammatory processes. HGF-1 and THP-1 cells were preincubated with specific inhibitors for MEK1/MEK2 (U0126), p38 (SB203580), NF- $\kappa$ B (PDTC), and JNK (SP600125) signaling, which resulted in a significant $(p<0.05)$ reduction of the $F$. alocis-induced COX2 expression for each of these inhibitors at $1 \mathrm{~d}$ (Figures $4(\mathrm{c})$ and $4(\mathrm{~d})$ ).

\section{Discussion}

Our study provides original evidence that the putative oral pathogen $F$. alocis is capable of inducing COX2 at transcriptional and protein levels in human fibroblastic and monocytic cells through TLR2 and MAPK signaling, suggesting a role of $F$. alocis in the etiopathogenesis of periodontal diseases. Interestingly, the stimulatory effect of $F$. alocis on COX2 was more pronounced in fibroblasts as compared to the monocytic cells, which emphasizes the significance of 


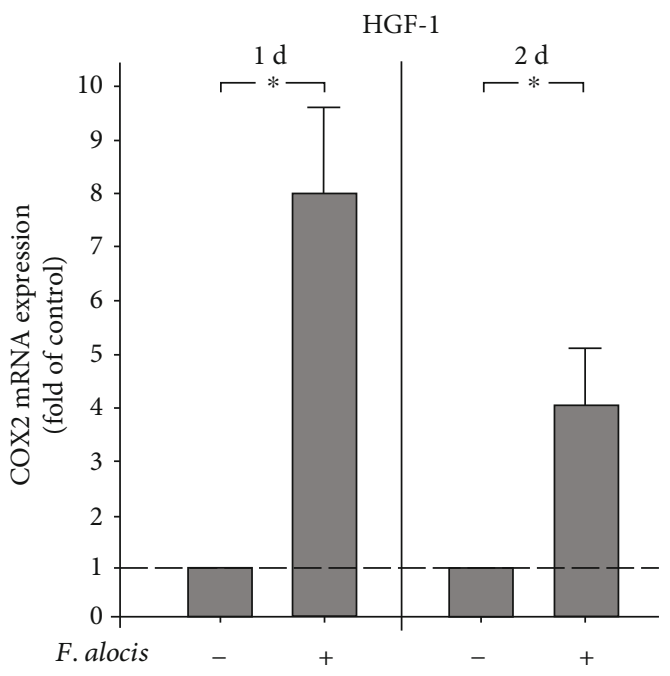

(a)

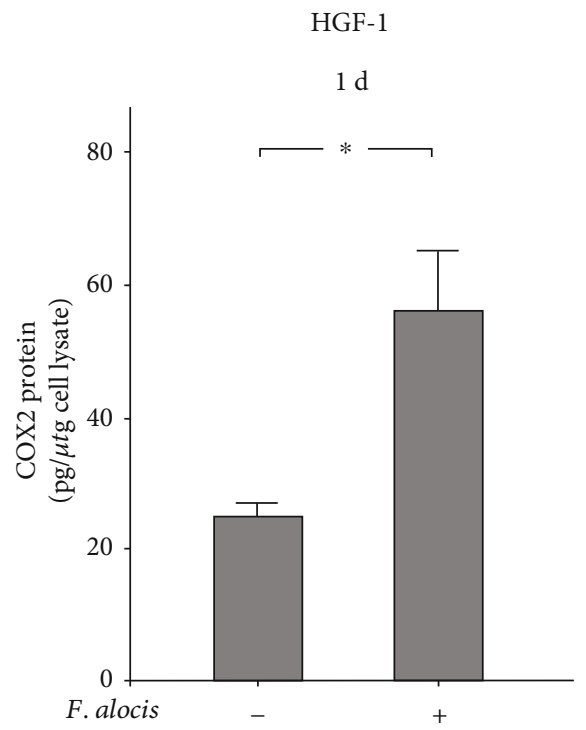

(c)

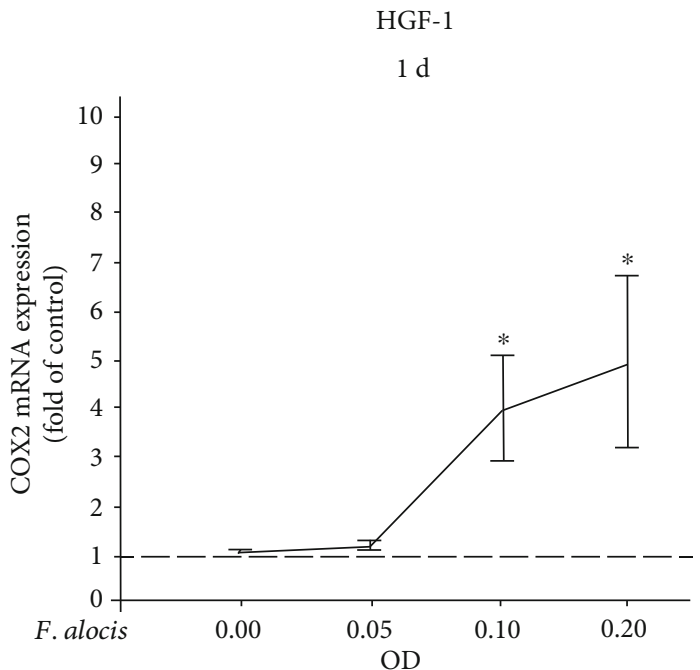

(b)

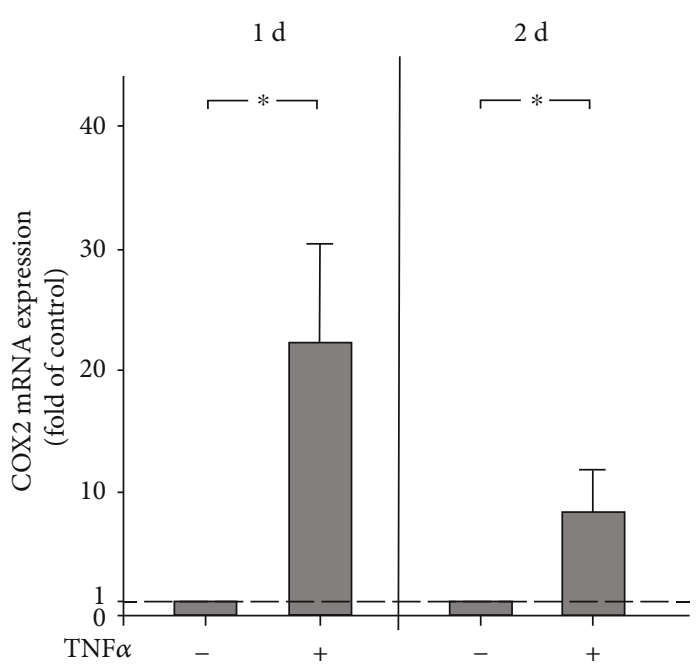

(d)

Figure 2: (a) Expression of COX2 in the presence and absence of F. alocis $\left(\mathrm{OD}_{660}: 0.1\right)$ in HGF-1 cells at $1 \mathrm{~d}$ and $2 \mathrm{~d}$, as analyzed by qPCR. Mean \pm SEM $(n=18)$. *Significant $(p<0.05)$ difference between groups. (b) Stimulation of COX2 expression by various concentrations of F. alocis $\left(\mathrm{OD}_{660}: 0.05,0.1\right.$, and 0.2$)$ in HGF-1 cells at $1 \mathrm{~d}$, as analyzed by qPCR. Unstimulated cells served as the control. Mean \pm SEM $(n=6) .{ }^{*}$ Significantly $(p<0.05)$ different from control. (c) COX2 protein level in lysates of HGF-1 cells in the presence and absence of F. alocis $\left(\mathrm{OD}_{660}: 0.1\right)$ at $1 \mathrm{~d}$, as analyzed by ELISA. Mean $\pm \operatorname{SEM}(n=12)$. ${ }^{*}$ Significant $(p<0.05)$ difference between groups. (d) Expression of COX2 in the presence and absence of TNF $\alpha(1 \mathrm{ng} / \mathrm{ml})$ in HGF- 1 cells at $1 \mathrm{~d}$ and $2 \mathrm{~d}$, as analyzed by qPCR. Mean \pm SEM $(n=26) .{ }^{*}$ Significant $(p<0.05)$ difference between groups.

periodontal fibroblasts as accessory inflammatory cells in periodontal inflammation.

The human oral microbiome comprises of more than 700 prokaryote species, and one of the periodontopathogens is $F$. alocis [24]. F. alocis is a Gram-positive, asaccharolytic, obligate anaerobic microorganism with a high prevalence in periodontal diseases [25]. Due to its virulence factors, F. alocis has the capacity to resist to oxidative stress, form biofilms, interact with other periodontopathogens, and produce proteases. Furthermore, periodontopathogens, such as F. alocis, are able to invade gingival cells and tissues, as the pocket epithelium is ulcerated in periodontitis, and can therefore get into the subepithelial blood vessels. As a consequence, infections with $F$. alocis result in the activation of the host immune response and, subsequently, the release of proinflammatory mediators by immunoinflammatory cells $[19,20,26]$. Ultimately, the dysregulated and excessive immunoinflammatory processes lead to soft tissue damage and alveolar bone resorption. COX2 is a key molecule of periodontal inflammation. Increased COX2 levels were found at sites of gingival or periodontal inflammation, and its expression has been shown to be associated with the severity of the disease $[10,11,27$. High COX2 levels in inflamed gingival tissues leads, in turn, to elevated PGE2 levels, which then further mediates the soft 


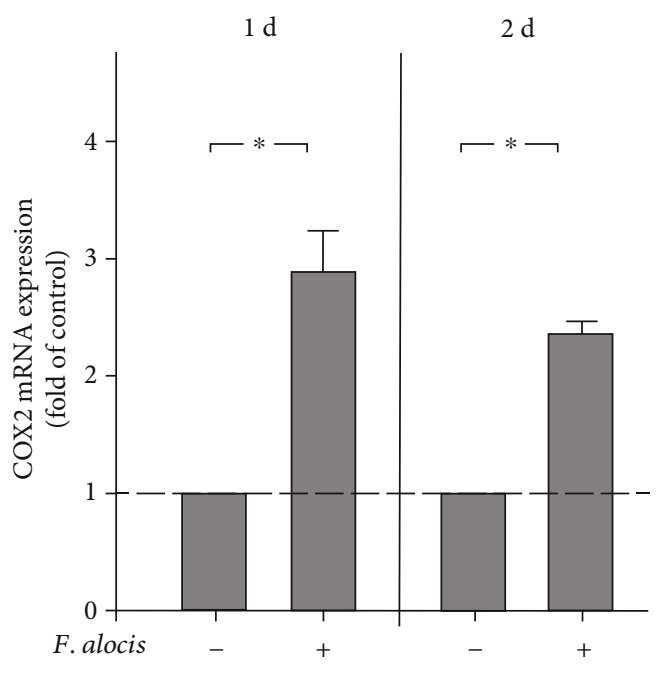

(a)

THP-1

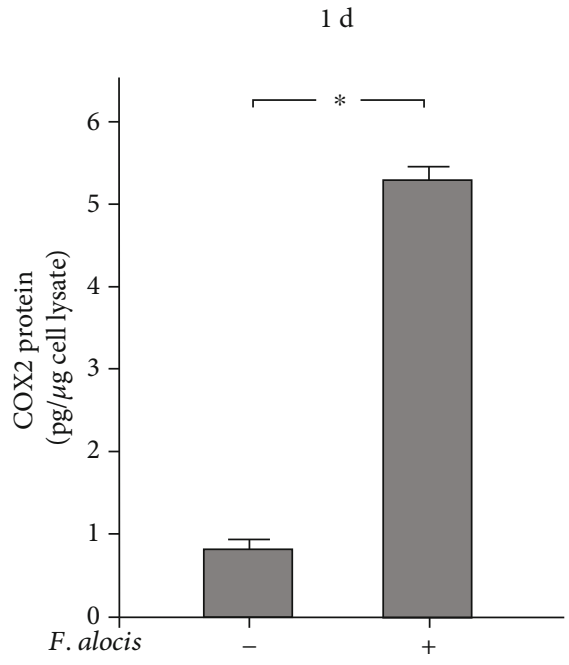

(c)
THP-1

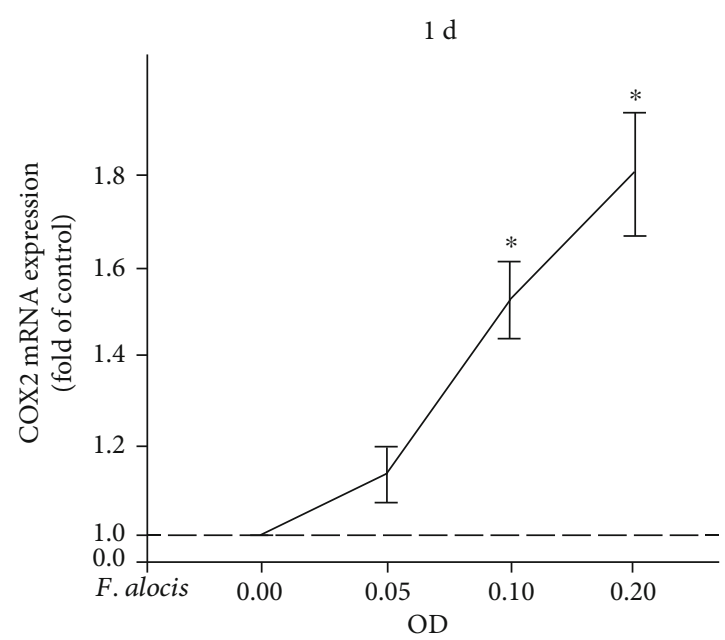

(b)

THP-1

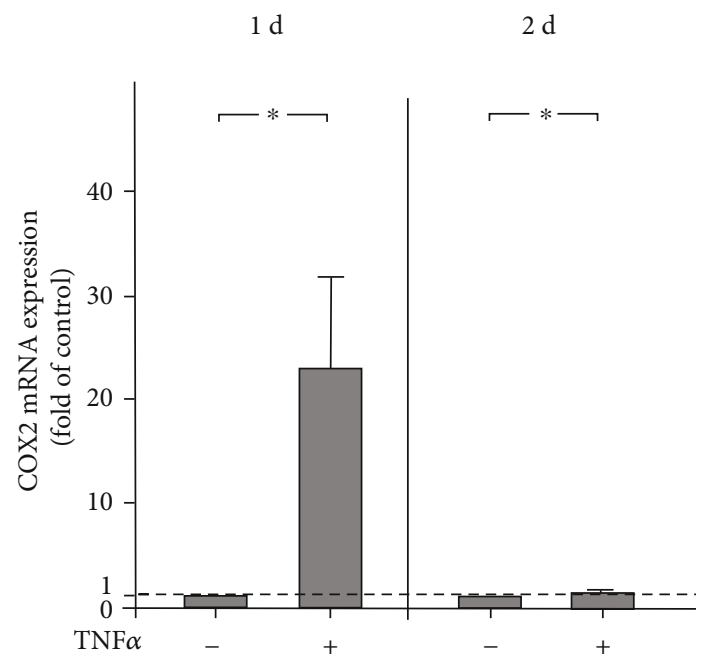

(d)

FIgURE 3: (a) Expression of COX2 in the presence and absence of F. alocis $\left(\mathrm{OD}_{660}: 0.1\right)$ in THP-1 cells at $1 \mathrm{~d}$ and $2 \mathrm{~d}$, as analyzed by qPCR. Mean \pm SEM $(n=18)$. *Significant $(p<0.05)$ difference between groups. (b) Stimulation of COX2 expression by various concentrations of F. alocis $\left(\mathrm{OD}_{660}: 0.05,0.1\right.$, and 0.2$)$ in THP-1 cells at $1 \mathrm{~d}$, as analyzed by qPCR. Unstimulated cells served as the control. Mean \pm SEM $(n=18) .{ }^{*}$ Significantly $(p<0.05)$ different from the control. (c) COX2 protein level in lysates of THP-1 cells in the presence and absence of $F$. alocis $\left(\mathrm{OD}_{660}: 0.1\right)$ at $1 \mathrm{~d}$, as analyzed by ELISA. Mean \pm SEM $(n=18)$. ${ }^{*}$ Significant $(p<0.05)$ difference between groups. (d) Expression of COX2 in the presence and absence of TNF $\alpha(1 \mathrm{ng} / \mathrm{ml})$ in THP-1 cells at $1 \mathrm{~d}$ and $2 \mathrm{~d}$, as analyzed by qPCR. Mean \pm SEM $(n=12) .{ }^{*}$ Significant $(p<0.05)$ difference between groups.

and hard tissue destruction [28], which highlights the importance of COX2 in the etiopathogenesis of periodontitis. Consequently, COX2 inhibitors have been evaluated for their adjunctive benefit in the treatment of periodontal diseases [29-31]. To date, no study has focused on the effect of F. alocis on the COX2 regulation, which could mediate, at least in part, the pathogenic actions of this microorganism on periodontal tissues. In this context, we sought to analyze what role structural resident cells of the periodontium, e.g., gingival fibroblasts, play as compared to professional inflammatory cells, e.g., monocytic cells.
Our data demonstrates for the first time that F. alocis upregulates the COX2 expression and increases the protein synthesis in both HGF-1 and THP-1 cells, suggesting that this putative pathogen also contributes to the elevated levels of COX2 in gingival tissues of periodontitis patients. Remarkably, the fibroblastic cells showed a more pronounced inflammatory response to $F$. alocis as compared to the monocytic cells, demonstrating the critical role of periodontal cells in the etiopathogenesis of periodontal diseases. It would be very interesting to also examine the effect of $F$. alocis on COX2 in gingival epithelial cells in future studies. 
HGF-1

$1 \mathrm{~d}$

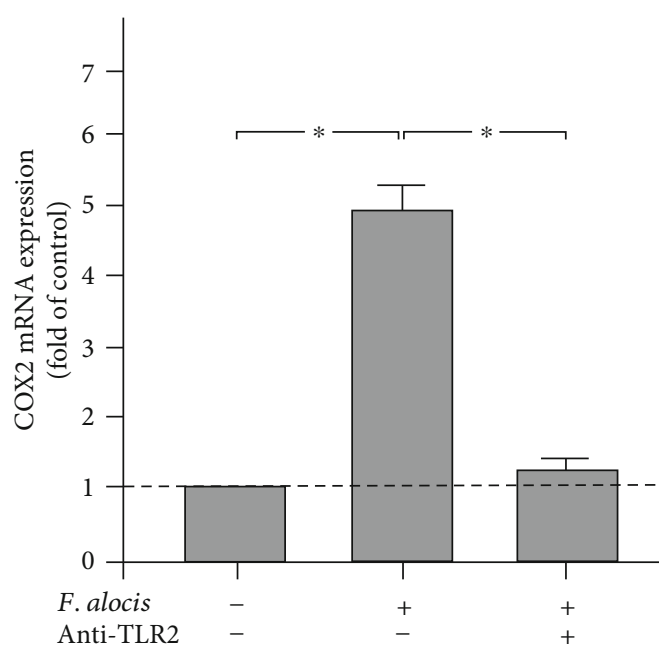

(a)

HGF-1

$1 \mathrm{~d}$
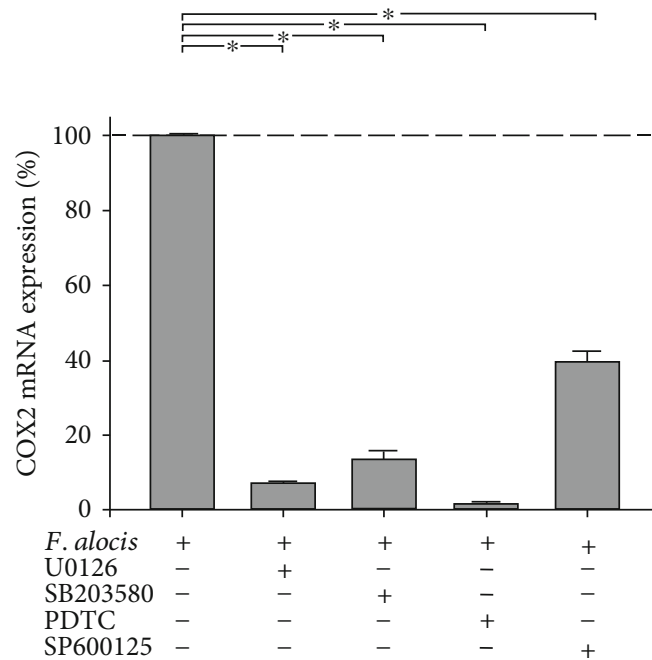

(c)
THP-1

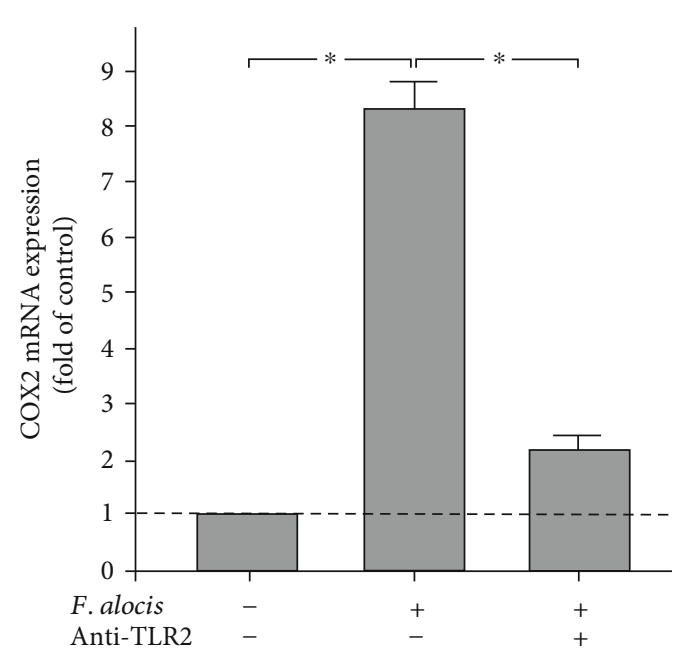

(b)

THP-1

$1 \mathrm{~d}$
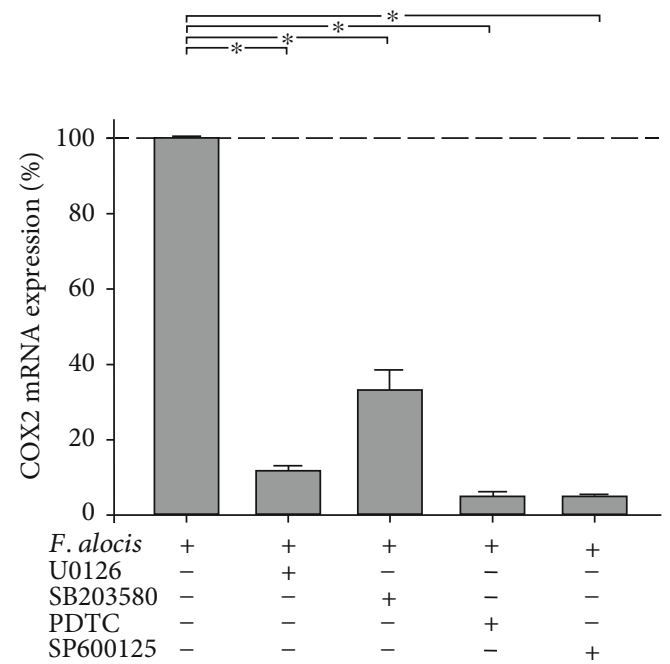

(d)

FIGURE 4: COX2 gene expression in response to F. alocis $\left(\mathrm{OD}_{660}: 0.1\right)$ in the presence and absence of anti-TLR2 blocking antibody in HGF-1 (a) and THP-1 (b) cells at $1 \mathrm{~d}$, as analyzed by qPCR. Mean \pm SEM $(n=3)$. * Significant $(p<0.05)$ difference between groups. COX2 gene expression in response to $F$. alocis $\left(\mathrm{OD}_{660}: 0.1\right)$ in the presence and absence of specific inhibitors against MEK1/MEK2 (U0126; 10 $\left.\mu \mathrm{M}\right)$, p38 (SB203580; $10 \mu \mathrm{M})$, NF- $\kappa$ B (PDTC; $10 \mu \mathrm{M})$, and JNK (SP600125; $10 \mu \mathrm{M})$ in HGF-1 (c) and THP-1 (d) cells at $1 \mathrm{~d}$, as analyzed by qPCR. Mean \pm SEM $(n=3) .{ }^{*}$ Significant $(p<0.05)$ difference between groups.

Interestingly, other investigators have observed that IL- $1 \beta$ and Aggregatibacter actinomycetemcomitans as well as Tannerella forsythia stimulate the COX2 expression in gingival fibroblasts, which is in agreement with our findings for TNF $\alpha$ and F. alocis [28]. We have previously demonstrated that Fusobacterium nucleatum is able to modulate COX2 levels in human periodontal ligament fibroblasts [32]. In summary, these observations suggest that periodontopathogens may use COX2 for their detrimental effects on periodontal cells and tissues.
Preincubation with a blocking TLR2 antibody resulted in a significant inhibition of the F. alocis-induced COX2 expression in HGF-1 and THP-1 cells, suggesting that $F$. alocis is interacting with this TLR for its inflammatory actions. Also, preincubation of HGF-1 and THP-1 cells with specific blockers against MAPK signaling, such as MEK1/MEK2, p38 MAPK, NF- $\kappa \mathrm{B}$, and JNK, caused an inhibition of the $F$. alocis-upregulated $\mathrm{COX} 2$ expression, indicating that these signaling pathways are involved in the regulation of COX2 by $F$. alocis in fibroblastic and monocytic cells. Recently, it 
has been demonstrated that interaction of $F$. alocis with neutrophils through TLR2 and subsequent activation of ERK1/2 and p38 MAPK pathways results in granule exocytosis as well as random and directed migration [16], which is in accordance with the observation that $F$. alocis exploits TLR2 and MAPKs for its effects. However, additional pathways could be involved in the actions of F. alocis on COX2 and should be studied in further investigations. In our experiments, a lysate of $F$. alocis was applied and the protease activity was still maintained. The used concentration of the bacterial lysate was also applied in previous studies on other periodontopathogens [32-34]. Although the knowledge on the F. alocis cell wall components is still limited, it could be assumed that lipoteichoic acid and/or peptidoglycan, which are present on the cell wall of Gram-positive bacteria, can directly stimulate fibroblasts or monocytes. Additional virulence factors of $F$. alocis could also have been contributed to the stimulatory effects of this microorganism on COX2 in our cells. Therefore, other receptors in addition to TLR2 may also have been implicated in the COX 2 regulation.

In order to better understand the pathogenic role of $F$. alocis in the etiopathogenesis of periodontal diseases, this periodontopathogen was selected for our study. The classical periodontopathogens $P$. gingivalis and $F$. nucleatum were also shown to upregulate COX2, which was used as a target in our study, in immunoinflammatory and periodontal fibroblastic cells, emphasizing again the key role of COX2 in the pathogenesis of periodontitis $[32,35]$. Since periodontitis is a polymicrobial disease, the effect of $F$. alocis in combination with other periodontopathogens should also be considered in future studies.

In one of our previous and recent study, we have used similar methodology but different experiments to evaluate the effect of $F$. alocis on the regulation of the collagenase MMP-1 in HGF-1 and THP-1 cells. We have demonstrated for the first time that $F$. alocis significantly increases the expression and protein synthesis of MMP-1 in both HGF-1 and THP-1 cells [21]. Interestingly, our results show original evidence that $F$. alocis may contribute to periodontal destruction through MMP-1 and to periodontal inflammation through COX2, demonstrating its role in two different stages of the periodontal disease process.

In summary, our in vitro study provides novel evidence that $F$. alocis upregulates COX2 at both transcriptional and protein levels through TLR2 and MAPK signaling mechanisms in human gingival fibroblastic and monocytic cells, suggesting that $F$. alocis may play a role in periodontal disease initiation and progression, therefore contributing to periodontal inflammation and tissue destruction. Moreover, the stimulatory action of F. alocis on COX2 was more pronounced in gingival fibroblasts as compared to the monocytic cells, underlining the critical role of these fibroblasts as accessory inflammatory cells in periodontal inflammation.

\section{Data Availability}

The data used to support the findings of this study are available from the corresponding author upon request.

\section{Conflicts of Interest}

The authors declare that there is no conflict of interests regarding the publication of this paper.

\section{Authors' Contributions}

Marjan Nokhbehsaim and Andressa V. B. Nogueira are the authors who contributed equally to this work.

\section{Acknowledgments}

This study was supported by the Medical Faculty of the University of Bonn. The authors would like to sincerely thank Ms. Ramona Menden and Ms. Silke van Dyck for their very valuable support.

\section{References}

[1] P. N. Papapanou, M. Sanz, N. Buduneli et al., "Periodontitis: consensus report of workgroup 2 of the 2017 world workshop on the classification of periodontal and peri-implant diseases and conditions," Journal of Periodontology, vol. 89, Supplement 1, pp. S173-S182, 2018.

[2] G. Hajishengallis and R. J. Lamont, "Beyond the red complex and into more complexity: the polymicrobial synergy and dysbiosis (PSD) model of periodontal disease etiology," Molecular Oral Microbiology, vol. 27, no. 6, pp. 409-419, 2012.

[3] J. L. Ebersole, D. Dawson III, P. Emecen-Huja et al., "The periodontal war: microbes and immunity," Periodontol 2000, vol. 75, no. 1, pp. 52-115, 2017.

[4] N. Zidar, K. Odar, D. Glavac, M. Jerse, T. Zupanc, and D. Stajer, "Cyclooxygenase in normal human tissues - is COX-1 really a constitutive isoform, and COX-2 an inducible isoform?," Journal of Cellular and Molecular Medicine, vol. 13, no. 9B, pp. 3753-3763, 2009.

[5] E. Ricciotti and G. A. FitzGerald, "Prostaglandins and inflammation," Arteriosclerosis, Thrombosis, and Vascular Biology, vol. 31, no. 5, pp. 986-1000, 2011.

[6] H. Hikiji, T. Takato, T. Shimizu, and S. Ishii, "The roles of prostanoids, leukotrienes, and platelet-activating factor in bone metabolism and disease," Progress in Lipid Research, vol. 47, no. 2, pp. 107-126, 2008.

[7] K. Noguchi and I. Ishikawa, "The roles of cyclooxygenase-2 and prostaglandin $\mathrm{E}_{2}$ in periodontal disease," Periodontol 2000, vol. 43, no. 1, pp. 85-101, 2007.

[8] P. Gümüş, N. Nizam, A. Nalbantsoy, Ö. Özçaka, and N. Buduneli, "Saliva, serum levels of interleukin-21, -33 and prostaglandin E2 in patients with generalised aggressive or chronic periodontitis," Oral Health \& Preventive Dentistry, vol. 15, no. 4, pp. 385-390, 2017.

[9] Ş. Kurgan, Ö. Fentoğlu, C. Önder et al., "The effects of periodontal therapy on gingival crevicular fluid matrix metalloproteinase-8, interleukin-6 and prostaglandin $\mathrm{E}_{2}$ levels in patients with rheumatoid arthritis," Journal of Periodontal Research, vol. 51, no. 5, pp. 586-595, 2016.

[10] L. Lazăr, A. Loghin, E. S. Bud, D. Cerghizan, E. Horváth, and E. E. Nagy, "Cyclooxygenase-2 and matrix metalloproteinase- 9 expressions correlate with tissue inflammation degree in periodontal disease," Romanian Journal of Morphology and Embryology, vol. 56, no. 4, pp. 1441-1446, 2015. 
[11] F. Mesa, F. O’Valle, M. Rizzo et al., "Association between COX-2 rs 6681231 genotype and interleukin- 6 in periodontal connective tissue. A pilot study," PLoS One, vol. 9, no. 2, article e87023, 2014.

[12] A. K. Kumar, N. R. Reddy, M. Babu, P. M. Kumar, V. S. Reddy, and C. V. Chavan, "Estimation of prostaglandin $\mathrm{E}_{2}$ levels in gingival crevicular fluid in periodontal health, disease and after treatment," Contemporary Clinical Dentistry, vol. 4, no. 3, pp. 303-306, 2013.

[13] G. A. Sánchez, V. A. Miozza, A. Delgado, and L. Busch, "Salivary IL-1 $\beta$ and PGE2 as biomarkers of periodontal status, before and after periodontal treatment," Journal of Clinical Periodontology, vol. 40, no. 12, pp. 1112-1117, 2013.

[14] C. Gonçalves, G. M. Soares, M. Faveri et al., "Association of three putative periodontal pathogens with chronic periodontitis in Brazilian subjects," Journal of Applied Oral Science, vol. 24, no. 2, pp. 181-185, 2016.

[15] H. Chen, Y. Liu, M. Zhang et al., “A Filifactor alocis-centered co-occurrence group associates with periodontitis across different oral habitats," Scientific Reports, vol. 5, p. 9053, 2015.

[16] C. L. Armstrong, I. Miralda, A. C. Neff et al., "Filifactor alocis promotes neutrophil degranulation and chemotactic activity," Infection and Immunity, vol. 84, no. 12, pp. 3423-3433, 2016.

[17] D. Belstrøm, N. E. Fiehn, C. H. Nielsen et al., "Differences in bacterial saliva profile between periodontitis patients and a control cohort," Journal of Clinical Periodontology, vol. 41, no. 2, pp. 104-112, 2014.

[18] A. P. Colombo, S. Bennet, S. L. Cotton et al., "Impact of periodontal therapy on the subgingival microbiota of severe periodontitis: comparison between good responders and individuals with refractory periodontitis using the human oral microbe identification microarray," Journal of Periodontology, vol. 83, no. 10, pp. 1279-1287, 2012.

[19] A. W. Aruni, F. Roy, and H. M. Fletcher, "Filifactor alocis has virulence attributes that can enhance its persistence under oxidative stress conditions and mediate invasion of epithelial cells by Porphyromonas gingivalis," Infection and Immunity, vol. 79, no. 10, pp. 3872-3886, 2011.

[20] C. E. Moffatt, S. E. Whitmore, A. L. Griffen, E. J. Leys, and R. J. Lamont, "Filifactor alocis interactions with gingival epithelial cells," Molecular Oral Microbiology, vol. 26, no. 6, pp. 365373, 2011.

[21] M. Nokhbehsaim, A. V. B. Nogueira, A. Damanaki et al., "Regulation of matrix metalloproteinase- 1 by Filifactor alocis in human gingival and monocytic cells," Clinical Oral Investigations, pp. 1-9, 2019.

[22] D. F. Madureira, I. Lucas de Abreu Lima, G. C. Costa, E. M. B. Lages, C. C. Martins, and T. Aparecida da Silva, "tumor necrosis factor-alpha in gingival crevicular fluid as a diagnostic marker for periodontal diseases: a systematic review," The Journal of Evidence-Based Dental Practice, vol. 18, no. 4, pp. 315-331, 2018.

[23] C. Ijuin, S. Ohno, K. Tanimoto, K. Honda, and K. Tanne, "Regulation of hyaluronan synthase gene expression in human periodontal ligament cells by tumour necrosis factor-alpha, interleukin-1beta and interferon-gamma," Archives of Oral Biology, vol. 46, no. 8, pp. 767-772, 2001.

[24] L. Gao, T. Xu, G. Huang, S. Jiang, Y. Gu, and F. Chen, "Oral microbiomes: more and more importance in oral cavity and whole body," Protein \& Cell, vol. 9, no. 5, pp. 488500, 2018 .
[25] W. Aruni, O. Chioma, and H. M. Fletcher, "Filifactor alocis: the newly discovered kid on the block with special talents," Journal of Dental Research, vol. 93, no. 8, pp. 725-732, 2014.

[26] A. W. Aruni, A. Mishra, Y. Dou, O. Chioma, B. N. Hamilton, and H. M. Fletcher, "Filifactor alocis - a new emerging periodontal pathogen," Microbes and Infection, vol. 17, no. 7, pp. 517-530, 2015.

[27] M. Miyauchi, M. Hiraoka, H. Oka et al., "Immuno-localization of COX-1 and COX-2 in the rat molar periodontal tissue after topical application of lipopolysaccharide," Archives of Oral Biology, vol. 49, no. 9, pp. 739-746, 2004.

[28] R. S. Morton and A. I. Dongari-Bagtzoglou, "Cyclooxygenase2 is upregulated in inflamed gingival tissues," Journal of Periodontology, vol. 72, no. 4, pp. 461-469, 2001.

[29] P. M. Preshaw, "Host modulation therapy with antiinflammatory agents," Periodontol 2000, vol. 76 , no. 1 , pp. 131-149, 2018.

[30] G. E. Salvi and N. P. Lang, "Host response modulation in the management of periodontal diseases," Journal of Clinical Periodontology, vol. 32, Supplement 6, pp. 108-129, 2005.

[31] N. Buduneli, S. Vardar, G. Atilla, T. Sorsa, H. Luoto, and H. Baylas, "Gingival crevicular fluid matrix metalloproteinase8 levels following adjunctive use of meloxicam and initial phase of periodontal therapy," Journal of Periodontology, vol. 73, no. 1, pp. 103-109, 2002.

[32] A. V. Nogueira, M. Nokhbehsaim, S. Eick et al., "Regulation of visfatin by microbial and biomechanical signals in PDL cells," Clinical Oral Investigations, vol. 18, no. 1, pp. 171-178, 2014.

[33] M. Nokhbehsaim, S. Keser, A. V. Nogueira et al., "Beneficial effects of adiponectin on periodontal ligament cells under normal and regenerative conditions," Journal Diabetes Research, vol. 2014, article 796565, 11 pages, 2014.

[34] A. Damanaki, M. Nokhbehsaim, S. Eick et al., "Regulation of NAMPT in human gingival fibroblasts and biopsies," Mediators of Inflammation, vol. 2014, Article ID 912821, 10 pages, 2014.

[35] Y. Murakami, A. Kawata, Y. Seki et al., "Comparative inhibitory effects of magnolol, honokiol, eugenol and bis-eugenol on cyclooxygenase-2 expression and nuclear factor-kappa B activation in RAW264.7 macrophage-like cells stimulated with fimbriae of Porphyromonas gingivalis," In Vivo, vol. 26, no. 6, pp. 941-950, 2012. 